\title{
Catherine M. Jones, Philippe de Vigneulles and the Art of Prose Translation
}

\section{Maria Colombo Timelli}

\section{(2) OpenEdition}

1 Journals

\section{Édition électronique}

URL : http://journals.openedition.org/studifrancesi/6227

DOI : 10.4000/studifrancesi.6227

ISSN : 2427-5856

Éditeur

Rosenberg \& Sellier

\section{Édition imprimée}

Date de publication : 1 novembre 2010

Pagination : 538

ISSN : 0039-2944

\section{Référence électronique}

Maria Colombo Timelli, "Catherine M. Jones, Philippe de Vigneulles and the Art of Prose Translation», Studi Francesi [En ligne], 162 (LIV | III) | 2010, mis en ligne le 30 novembre 2015, consulté le 07 janvier 2021. URL : http://journals.openedition.org/studifrancesi/6227 ; DOI : https://doi.org/10.4000/ studifrancesi.6227

Ce document a été généré automatiquement le 7 janvier 2021.

\section{(c)}

Studi Francesi è distribuita con Licenza Creative Commons Attribuzione - Non commerciale - Non opere derivate 4.0 Internazionale. 


\title{
Catherine M. Jones, Philippe de Vigneulles and the Art of Prose Translation
}

\author{
Maria Colombo Timelli
}

\section{RÉFÉRENCE}

CATHERINE M. JONES, Philippe de Vigneulles and the Art of Prose Translation, Cambridge, D.S.

Brewer, 2008, pp. 151.

1 Ce livre constitue la première monographie consacrée à Philippe de Vigneulles: le sujet traité par C.M.J. va en effet bien au-delà de ce qu'annonce le titre, puisque la 'mise en prose' du cycle des Lorrains y est constamment rapportée à l'ensemble de l'œuvre de l'auteur messin.

2 Philippe de Vigneulles peut être considéré comme un auteur médiéval 'tardif', à l'instar de son contemporain lyonnais Pierre Sala, ce qui lui a valu jusqu'ici un certain mépris tant de la part des médiévistes (avec les notables exceptions de Pierre Demarolle et de Jean-Charles Herbin) que de la part des seiziémistes; de surcroît, profondément ancré dans l'histoire de sa ville et de sa région, il a certes intéressé les historiens locaux, mais sans vraiment gagner l'attention de la critique. Auteur d'une Chronique de Metz (1525), d'un Journal riche en informations locales (1522), d'un recueil de 110 Nouvelles (terminé après 1515), Philippe a encore remanié en prose le cycle des Lorrains (Hervis de Metz, Garin le Lorrain, Gerbert de Metz, Anseis de Metz), adaptation qui fait l'objet principal, mais non pas le seul - comme on l'a dit - de ce volume. La lecture de cet ensemble épique n'est pas aisée: le manuscrit autrefois conservé à Metz (B.M. 847, ms. v) ayant brûlé en 1944, il n'en subsiste qu'un microfilm de la MLA; le manuscrit Hunolstein ( $h$, provisoirement conservé auprès de la Parker Library, Corpus Christi College, Cambridge), illustré de la main de l'auteur, transmet une rédaction révisée, qui intègre justement des corrections de $v$, mais n'est toujours pas édité, ce qui fait de l'étude de 
Mme Jones un travail de première main. Sa perspective est opportunément centrée sur la prose elle-même, considérée non pas comme la réécriture tardive et délayée d'une épopée 'classique', mais pour ce qu'elle ajoute à cette source et qui témoigne par conséquent des préoccupations esthétiques, sociales, géopolitiques de l'auteur; j'hésiterais cependant à franchir le pas successif, ce que fait C.M.J. p.12, lorsqu'elle affirme que ces préoccupations sont aussi celles du public de Philippe de Vigneulles: ceci reste vraisemblable, mais on ne saurait pas oublier que, resté à l'état de manuscrit, son texte n'a de fait pas véritablement circulé. En dehors de tout patronage, en effet, Philippe n'a ni prétentions savantes ni orgueil d'auteur, mais élabore dans toute son œuvre un véritable projet civique: la célébration de sa ville à un moment critique de son histoire, où sa liberté même était en danger. C'est pourquoi Mme Jones situe les Lorrains en prose au centre d'un double réseau intertextuel: d'une part, une intertextualité 'interne', par rapport aux autres œuvres de Philippe, et une 'externe', avec les deux autres adaptations tardives des Lorrains ( $\mathrm{xv}^{\mathrm{e}}$ siècle), à savoir la Prose dite 'de l'Arsenal' (ms. 3346, éd. par JeanCharles Herbin en 1995), et l'Histoire de Charles Martel en prose, élaborée en milieu bourguignon (un ms. copié par David Aubert, éd. partielle par Valérie Naudet en 2005) (Introduction, pp. 1-16).

3 L'étude de C.M.J. est organisée en trois parties. Un chapitre («Truth, Translation and the Medium of Prose», pp.17-41) est consacré à la conception de la transcription en prose chez Philippe de Vigneulles; une analyse attentive du prologue fait ressortir sa réflexion sur la 'vérité' et l'autorité' du texte, ainsi qu'une conscience aigüe des questions linguistiques, sensibilité que confirment tant le Journal que les Nouvelles. Le rôle dévolu à la réécriture en prose coïncide par ailleurs dans les Lorrains avec les nécessités d'abréviation et de concision.

4 Le chapitre successif («Excellent Stories, Marvelous Deeds and the Prosaics of Crisis», pp. 42-85) porte plus précisément sur le traitement du matériau narratif, les choix effectués, l'insertion de la légende épique dans les préoccupations civiques, personnelles et littéraires de l'auteur. Philippe privilégie sans aucun doute la chronique urbaine, célèbre les héros locaux (Garin au premier chef), adapte le contenu à la mentalité bourgeoise et marchande de sa ville et de son temps.

Un dernier chapitre («The Craft of 'dérimage'», pp. 86-128) concerne plus précisément la voix du 'translateur' et sa présence dans le texte: grâce aux ajouts, abréviations, pratiques de composition, interventions autoriales, relevés par C.M.J., le genre épique, encore populaire à l'extrême fin du Moyen Âge, est mis au service de la gloire locale.

Comme le souligne la «Conclusion» (pp. 129-133), la prose de Philippe, toute centrée sur l'identité culturelle et civique d'une ville en crise, marque la fin de la 'première vague' dans l'histoire des traductions intralinguistiques de la Geste des Lorrains, avant le médiévalisme renouvelé du XIX ${ }^{\mathrm{e}}$ siècle, autre moment critique dans l'histoire de Metz et de la Lorraine. Une bibliographie importante (sources primaires et bibliographie critique) est réunie aux pp. 149-151.

Nous avons là un beau livre, susceptible d'intéresser non seulement les spécialistes de Philippe de Vigneulles - ils ne sont pas légion - et de la réception de la matière épique, mais plus en général tous les critiques qui s'occupent des réécritures en prose - et ceuxci sont bien plus nombreux depuis quelques années - entre la fin du Moyen Âge et le siècle de la Renaissance. 